\title{
GLOBAL REGULARITY FOR GENERAL NON-LINEAR WAVE EQUATIONS I. $(6+1)$ AND HIGHER DIMENSIONS
}

\author{
JACOB STERBENZ
}

\begin{abstract}
Following work of Tataru, [15] and [13], we solve the division problem for wave equations with generic quadratic non-linearities in high dimensions. Specifically, we show that non-linear wave equations which can be written as systems involving equations of the form $\square \phi=\phi \nabla \phi$ and $\square \phi=|\nabla \phi|^{2}$ are well-posed with scattering in $(6+1)$ and higher dimensions if the Cauchy data are small in the scale invariant $\ell^{1}$ Besov space $\dot{B}^{s_{c}, 1}$. This paper is the first in a series of works where we discuss the global regularity properties of general non-linear wave equations for all dimensions $4 \leqslant n$.
\end{abstract}

\section{INTRODUCTION}

In this paper, our aim is to give a more or less complete description of the global regularity properties of generic homogeneous quadratic non-linear wave equations on $(6+1)$ and higher dimensional Minkowski space. The equations we will consider are all of the form:

$$
\square \phi=\mathcal{N}(\phi, D \phi) .
$$

Here $\square:=-\partial_{t}^{2}+\Delta_{x}$ denotes the standard wave operator on $\mathbb{R}^{n+1}$, and $\mathcal{N}$ is a smooth function of $\phi$ and its first partial derivatives, which we denote by $D \phi$. For all of the nonlinearities we study here, $\mathcal{N}$ will be assumed to be at least quadratic in nature, that is:

$$
\mathcal{N}(X, Y)=O\left(|(X, Y)|^{2}\right), \quad(X, Y) \sim 0 .
$$

The homogeneity condition we require $\mathcal{N}$ to satisfy is that there exist a (vector) $\sigma$ such that:

$$
\mathcal{N}\left(\lambda^{\sigma} \phi, \lambda^{\sigma+1} D \phi\right)=\lambda^{\sigma+2} \mathcal{N}(\phi, D \phi),
$$

where we use multiindex notation for vector $\mathcal{N}$. The condition (2) implies that solutions to the system (11) are invariant (again solutions) if one performs the scale transformations:

$$
\phi(\cdot) \rightsquigarrow \lambda^{\sigma} \phi(\lambda \cdot) .
$$

The general class of equations which falls under this description contains virtually all massless non-linear field theories on Minkowski space, including the Yang Mills equations (YM), the wave-maps equations (WM), and the Maxwell-Dirac equations (MD). We list the schematics for these systems respectively as:

This work was conducted under NSF grant DMS-0100406. 


$$
\begin{aligned}
\square \phi & =|D \phi|^{2}, \\
\square u & =A D u, \\
\square A & =|D u|^{2} .
\end{aligned}
$$

The various values of $\sigma$ for these equations are (respectively) $\sigma=1, \sigma=0$, and $\sigma=\left(\frac{1}{2}, 1\right)$. For a more complete introduction to these equations, see for instance the work [2] and [1. For the purposes of this paper, will will only be concerned with the structure of these equations at the level of the generic schematics (YM)-(MD).

The central problem we will be concerned with is that of giving a precise description of the regularity assumptions needed in order to guarantee that the Cauchy problem for the system (1) is globally well posed with scattering (GWPS). That is, given initial data:

$$
\phi(0)=f, \quad \partial_{t} \phi(0)=g,
$$

we wish to describe how much smoothness and decay $(f, g)$ needs to possess in order for there to exist a unique global solution to the system (1) with this given initial data. We also wish to show that the solutions we construct depend continuously on the initial data, and are asymptotic to solutions of the linear part of (1). We will describe shortly in what sense we will require these notions to hold.

Our main motivation here is to be able to prove global well-posedness for nonlinear wave equations of the form (1) in a context where the initial data may not be very smooth, and furthermore does not possess enough decay at space-like infinity to be in $L^{2}$. Also, we would like to understand how this can be done in situations where the equations being considered contain no special structure in the non-linearity. For instance, this is of interest in discussing the problem of small data global well-posedness for the Maxwell-Klein-Gordon and Yang-Mills equations with the Lorentz gauge enforced instead of the more regular Coulomb gauge. This provides a significant point of departure from earlier works on the global existence theory of non-linear wave equations, which for the general case requires precise control on the initial data in certain weighted Sobolev spaces (see 4]), or else requires the non-linearity to have some special algebraic or gauge structure which allows one to exploit some null form identities or apply an appropriate renormalization to the equation being considered (see [13] and [12]).

From the point of view of homogeneity, we are lead directly to considerations of the low regularity properties of the equations (11) as follows: By a simple scaling argument ${ }^{1}$ : one can see that the most efficient $L^{2}$ based regularity assumption

\footnotetext{
${ }^{1}$ In conjunction with finite time blowup for large data. This phenomena is known to happen for higher dimensional equations with derivative non-linearities even in the presence of positive conserved quantities.
} 
possible on the initial data involves $s_{c}=\frac{n}{2}-\sigma$ derivatives. Again, by scale invariance and looking at unit frequency initial $\mathrm{data}^{2}$, one can see that if we are to impose only an $L^{2}$ smallness condition on the initial data which contains no physical space weights, then $s_{c}=\frac{n}{2}-\sigma$ is in fact the largest amount of derivatives we may work with. This leads us to consider the question of GWPS for initial data in the homogeneous Besov spaces $\dot{B}^{s_{c}, p}$, for various values of $p$. In this work, we will concentrate solely on the case $p=1$. This is the strongest scale and translation invariant control on the initial data possible, and will be crucial for the kind of non-linearities we work with here. In fact, it does not seem possible to push any type of global regularity for equations of the type (11) which contain derivatives in the non-linearity down to the scale invariant Sobolev space $\dot{H}^{s_{c}}=\dot{B}^{s_{c}, 2}$ unless the equations under consideration possess a great deal of special structure in the non-linearity. This has been done for the wave-maps equations (see 12) and more recently for the Maxwell-Klein-Gordon equations in $(6+1)$ and higher dimensions with the help of the Coulomb gauge (see [11]).

In recent years, there has been much progress in our understanding of the low regularity local theory for general non-linear wave equations of the form (11). In the lower dimensional setting, i.e. when $n=2,3,4$, it is known from counterexamples of Lindblad (see [10]) that there is ill posedness for initial data in the Sobolev space $H^{s_{0}}$, where $s_{0} \leqslant s_{c}+\frac{5-n}{4}$. Intimately connected with this phenomena is the failure of certain space-time estimates for the linear wave equation known as Strichartz estimates. Specifically, one does not have anything close to an $L^{2}\left(L^{4}\right)$ estimate in these dimensions. Such an estimate obviously plays a crucial role (via Duhamel's principle) in the quadratic theory. However, using the Strichartz estimates available in these dimensions along with Picard iteration in certain function spaces, one can show that the Lindblad counterexamples are sharp in there is local well-posedness for initial data in the spaces $H^{s}$ when $s_{c}+\frac{5-n}{4}<s$ (see for example [9]).

In the higher dimensional setting, i.e. when the number of spatial dimensions is $n=5$ or greater, one does have access to Strichartz estimates at the level of $L^{2}\left(L^{4}\right)$ (see [3]), and it is possible to push the local theory down to $H^{s_{c}+\epsilon}$, where $0<\epsilon$ is arbitrary (see [15]).

In all dimensions, the single most important factor which determines the local theory as well as the range of validity for Strichartz estimates is the existence of free waves which are highly concentrated along null directions in Minkowski space. These waves, known as Knapp counterexamples, resemble a single beam of light which remains coherent for a long period of time before dispersing. For a special class of non-linearities, known as "null structures", interactions between these coherent beams are effectively canceled, and one gains an improvement in the local theory of equations whose nonlinearities have this form (see for example [5], 9]).

In both high and low dimensional settings, the analysis of certain null structures, specifically non-linearities containing the $Q_{0}$ null from ${ }^{3}$, has led to the proof

\footnotetext{
${ }^{2}$ That is initial data sets where the Fourier transform is supported in the unit frequency annulus $\left\{\xi\left|\frac{1}{2}<\right| \xi \mid<2\right\}$.

${ }^{3}$ This is defined by the equation $Q_{0}(\phi, \psi)=\partial_{\alpha} \phi \partial^{\alpha} \psi$.
} 
that the wave-maps model equations ${ }^{4}$ are well posed in the scale invariant $\ell^{1}$ Besov space $\dot{B}^{\frac{n}{2}, 1}$ (see [13] and 14]). While the proof of this result is quite simple for high dimensions, it relies in an essential way on the structure of the $Q_{0}$ null form. In fact, there is no direct way to extend the proof of this result to include the less regular nonlinearities of the form $\phi \nabla \phi$, or for that matter the $Q_{i j}$ null forms ${ }^{5}$, which show up in the equations of gauge field theory. However, the high dimensional non-linear interaction of coherent waves is quite weak (e.g. giving the desired range of validity for Strichartz estimates), and one would expect that it is possible to prove local well posedness for quadratic equations with initial data in the scale invariant $\ell^{1}$ Besov space without resorting to any additional structure in the nonlinearity. For $n=5$ dimensions, it may be that this is not quite possible, although we provide no convincing evidence except for the fact that there is no obvious way to add over our localized estimates in that dimension in order to obtain a full set of estimates that works in all of space-time Fourier space. For $n=6$ and higher dimensions, we will prove that in fact no null structure is needed for there to be well posedness in $\dot{B}^{s_{c}, 1}$. This leads to the statement of our main result which is as follows:

Theorem 1.1 (Global Well Posedness). Let $6 \leqslant n$ be the number of spatial dimensions. For any of the generic equations listed above: $Y M, W M$, or $M D$, let $(f, g)$ be a (possibly vector valued) initial data set. Let $s_{c}=\frac{n}{2}-\sigma$ be the corresponding $L^{2}$ scaling exponent. Then there exists constants $0<\epsilon_{0}, C$ such that if

$$
\|(f, g)\|_{\dot{B}^{s_{c}, 1} \times \dot{B}^{s_{c}-1,1}} \leqslant \epsilon_{0}
$$

there exits a global solution $\psi$ which satisfies the continuity condition:

$$
\|\psi\|_{C\left(\dot{B}^{s_{c}, 1}\right) \cap C^{(1)}\left(\dot{B}^{s_{c}-1,1}\right)} \leqslant C\|(f, g)\|_{\dot{B}^{s_{c}, 1} \times \dot{B}^{s_{c}-1,1}} .
$$

The solution $\psi$ is unique in the following sense: There exists a sequence of smooth functions $\left(f_{N}, g_{N}\right)$ such that:

$$
\lim _{N \rightarrow \infty}\left\|(f, g)-\left(f_{N}, g_{N}\right)\right\|_{\dot{B}^{s_{c}, 1} \times \dot{B}^{s_{c}-1,1}}=0 .
$$

For this sequence of functions, there exists a sequence of unique smooth global solutions $\psi_{N}$ of (1) with this initial data. Furthermore, the $\psi_{N}$ converge to $\psi$ as follows:

$$
\lim _{N \rightarrow \infty}\left\|\psi-\psi_{N}\right\|_{C\left(\dot{B}^{s_{c}, 1}\right) \cap C^{(1)}\left(\dot{B}^{s_{c}-1,1}\right)}=0
$$

Also, $\psi$ is the only solution which may be obtained as a limit (in the above sense) of solutions to (1) with regularizations of $(f, g)$ as initial data. Finally, $\psi$ retains any extra smoothness inherent in the initial data. That is, if $(f, g)$ also has finite $\dot{H}^{s} \times \dot{H}^{s-1}$ norm, for $s_{c}<s$, then so does $\psi$ at fixed time and one has the following estimate:

$$
\|\psi\|_{C\left(\dot{H}^{s}\right) \cap C^{(1)}\left(\dot{H}^{s-1}\right)} \leqslant C\|(f, g)\|_{\dot{H}^{s} \times \dot{H}^{s-1}}
$$

\footnotetext{
${ }^{4}$ Not the rough schematic we have listed here, but rather equations of the form $\square \phi=\Gamma(\phi) Q_{0}(\phi, \phi)$.

${ }^{5}$ These are defined by $Q_{i j}(\phi, \psi)=\partial_{i} \phi, \partial_{j} \psi-\partial_{j} \phi \partial_{i} \psi$.
} 
In a straightforward way, the function spaces we iterate in allow us to show the following scattering result without any extra work:

Theorem 1.2. Using the same notation as above we have that there exists data sets $\left(f^{ \pm}, g^{ \pm}\right)$, such that if $\psi^{ \pm}$is the solution to the homogeneous wave equation with the corresponding initial data, the following asymptotics hold:

$$
\begin{aligned}
\lim _{t \rightarrow \infty}\left\|\psi^{+}-\psi\right\|_{\dot{B}^{s_{c}, 1} \cap \partial_{t} \dot{B}^{s_{c}-1,1}} & =0, \\
\lim _{t \rightarrow-\infty}\left\|\psi^{-}-\psi\right\|_{\dot{B}^{s_{c}, 1} \cap \partial_{t} \dot{B}^{s_{c}-1,1}} & =0 .
\end{aligned}
$$

Furthermore, the scattering operator retains any additional regularity inherent in the initial data. That is, if $(f, g)$ has finite $\dot{H}^{s}$ norm, then so does $\left(f^{ \pm}, g^{ \pm}\right)$, and the following asymptotics hold:

$$
\begin{aligned}
\lim _{t \rightarrow \infty}\left\|\psi^{+}-\psi\right\|_{\dot{H}^{s} \cap \partial_{t} \dot{H}^{s-1}} & =0, \\
\lim _{t \rightarrow-\infty}\left\|\psi^{-}-\psi\right\|_{\dot{H}^{s} \cap \partial_{t} \dot{H}^{s-1}} & =0 .
\end{aligned}
$$

\section{Preliminary Notation}

For quantities $A$ and $B$, we denote by $A \lesssim B$ to mean that $A \leqslant C \cdot B$ for some large constant $C$. The constant $C$ may change from line to line, but will always remain fixed for any given instance where this notation appears. Likewise we use the notation $A \sim B$ to mean that $\frac{1}{C} \cdot B \leqslant A \leqslant C \cdot B$. We also use the notation $A \ll B$ to mean that $A \leqslant \frac{1}{C} \cdot B$ for some large constant $C$. This is the notation we will use throughout the paper to break down quantities into the standard cases: $A \sim B$, or $A \ll B$, or $B \ll A$; and $A \lesssim B$, or $B \ll A$, without ever discussing which constants we are using.

For a given function of two variables $(t, x) \in \mathbf{R} \times \mathbf{R}^{3}$ we write the spatial and space-time Fourier transform as:

$$
\begin{aligned}
& \widehat{f}(t, \xi)=\int e^{-2 \pi i \xi \cdot x} f(t, x) d x \\
& \widetilde{f}(\tau, \xi)=\int e^{-2 \pi i(\tau t+\xi \cdot x)} f(t, x) d t d x
\end{aligned}
$$

respectively. At times, we will also write $\mathcal{F}[f]=\widetilde{f}$.

For a given set of functions of the spatial variable only, we denote by $W(f, g)$ the solution of the homogeneous wave equation with Cauchy data $(f, g)$. If $F$ is a function on space-time, we will denote by $W(F)$ the function $W\left(F(0), \partial_{t} F(0)\right)$.

Let $E$ denote any fundamental solution to the homogeneous wave equation. i.e., one has the formula $\square E=\delta$. We define the standard Cauchy parametrix for the wave equation by the formula:

$$
\frac{1}{\square} F=E * F-W(E * F) .
$$


Explicitly, one has the identity:

$$
\widehat{\frac{1}{\square} F}(t, \xi)=-\int_{0}^{t} \frac{\sin (2 \pi|\xi|(t-s))}{2 \pi|\xi|} \widehat{F}(s, \xi) d s .
$$

For any function $F$ which is supported away from the light cone in Fourier space, we shall use the following notation for division by the symbol of the wave equation:

$$
\frac{1}{\Xi} F=E * F
$$

Of course, the definition of $\frac{1}{E}$ does not depend on $E$ so long as for $F$ is supported away from the light cone; for us that will always be the case when we use this notation. Explicitly, one has the formula:

$$
\mathcal{F}\left[\frac{1}{\Xi} F\right](\tau, \xi)=\frac{1}{4 \pi^{2}\left(\tau^{2}-|\xi|^{2}\right)} \widetilde{F}(\tau, \xi)
$$

\section{Multipliers and Function Spaces}

Let $\varphi$ be a smooth bump function (i.e. supported on the set $|s| \leqslant 2$ such that $\varphi=1$ for $|s| \leqslant 1$ ). In what follows, it will be a great convenience for us to assume that $\varphi$ may change its exact form for two separate instances of the symbol $\varphi$ (even if they occur on the same line). In this way, we may assume without loss of generality that in addition to being smooth, we also have the idempotence identity $\varphi^{2}=\varphi$. We shall use this convention for all the cutoff functions we introduce in the sequel.

For $\lambda \in 2^{\mathbb{Z}}$, we denote the dyadic scaling of $\varphi$ by $\varphi_{\lambda}(s)=\varphi\left(\frac{s}{\lambda}\right)$. The most basic Fourier localizations we shall use here are with respect to the space-time variable and the distance from the cone. Accordingly, we form the Littlewood-Paley type cutoff functions:

$$
\begin{aligned}
& s_{\lambda}(\tau, \xi)=\varphi_{2 \lambda}(|(\tau, \xi)|)-\varphi_{\frac{1}{2} \lambda}(|(\tau, \xi)|), \\
& c_{d}(\tau, \xi)=\varphi_{2 d}(|\tau|-|\xi|)-\varphi_{\frac{1}{2} d}(|\tau|-|\xi|) .
\end{aligned}
$$

We now denote the corresponding Fourier multiplier operator via the formulas $\widetilde{S_{\lambda} u}=s_{\lambda} \widetilde{u}$ and $\widetilde{C_{d} u}=c_{d} \widetilde{u}$ respectively. We also use a multi-subscript notation to denote products of the above operators, e.g. $S_{\lambda, d}=S_{\lambda} C_{d}$. We shall use the notation:

$$
S_{\lambda, \bullet \leqslant d}=\sum_{\delta \leqslant d} S_{\lambda, \delta}
$$

to denote cutoff in an $O(d)$ neighborhood of the light cone in Fourier space. At times it will also be convenient to write $S_{\lambda, d \leqslant \bullet}=S_{\lambda}-S_{\lambda, \bullet<d}$. We shall also use the notation $S_{\lambda, d}^{ \pm}$etc. to denote the multiplier $S_{\lambda, d}$ cutoff in the half space $\pm \tau>0$. 
The other type of Fourier localization which will be central to our analysis is the decomposition of the spatial variable into radially directed blocks of various sizes. To begin with, we denote the spatial frequency cutoff by:

$$
p_{\lambda}(\xi)=\varphi_{2 \lambda}(|\xi|)-\varphi_{\frac{1}{2} \lambda}(|\xi|),
$$

with $P_{\lambda}$ the corresponding operator. For a given parameter $\delta \leqslant \lambda$, we now decompose $P_{\lambda}$ radially as follows. First decompose the the unit sphere $S^{n-1} \subset \mathbb{R}^{n}$ into angular sectors of size $\frac{\delta}{\lambda} \times \ldots \times \frac{\delta}{\lambda} \quad$ with bounded overlap (independent of $\delta)$. These angular sectors are then projected out to frequency $\lambda$ via rays through the origin. The result is a decomposition of $\operatorname{supp}\left\{p_{\lambda}\right\}$ into radially directed blocks of size $\lambda \times \delta \times \ldots \times \delta$ with bounded overlap. We enumerate these blocks and label the corresponding partition of unity by $b_{\lambda, \delta}^{\omega}$. It is clear that things may be arranged so that upon rotation onto the $\xi_{1}$-axis, each $b_{\lambda, \delta}^{\omega}$ satisfies the bound:

$$
\left|\partial_{1}^{N} b_{\lambda, \delta}^{\omega}\right| \leqslant C_{N} \lambda^{-N}, \quad\left|\partial_{i}^{N} b_{\lambda, \delta}^{\omega}\right| \leqslant C_{N} \delta^{-N} .
$$

In particular, each $B_{\lambda, \delta}^{\omega}$ is given by convolution with an $L^{1}$ kernel. We shall also denote:

$$
S_{\lambda, d}^{\omega}=B_{\lambda,(\lambda d)^{\frac{1}{2}}}^{\omega} S_{\lambda, d}, \quad \quad S_{\lambda, \bullet \leqslant d}^{\omega}=B_{\lambda,(\lambda d)^{\frac{1}{2}}}^{\omega} S_{\lambda, \bullet \leqslant d}
$$

Note that the operators $S_{\lambda, d}^{\omega}$ and $S_{\lambda, \bullet \leqslant d}^{\omega}$ are only supported in the region where $|\tau| \lesssim|\xi|$

We now use these multipliers to define the following dyadic norms, which will be the building blocks for the function spaces we will use here.

$$
\begin{array}{ll}
\|u\|_{X_{\lambda, p}^{\frac{1}{2}}}^{p}=\sum_{d \in 2^{\mathbb{Z}}} d^{\frac{p}{2}}\left\|S_{\lambda, d} u\right\|_{L^{2}}^{p}, & \text { ("classical" } H^{s, \delta} \text { ) } \\
\|u\|_{Y_{\lambda}}=\lambda^{-1}\left\|\square S_{\lambda} u\right\|_{L^{1}\left(L^{2}\right)} & \text { (Duhamel) } \\
\|u\|_{Z_{\lambda}}=\lambda^{\frac{2-n}{2}} \sum_{d}\left(\sum_{\omega}\left\|S_{\lambda, d}^{\omega} u\right\|_{L^{1}\left(L^{\infty}\right)}^{2}\right)^{\frac{1}{2}} . & \text { (outer block) }
\end{array}
$$

Notice that the (semi) norms $X_{\lambda, p}^{\frac{1}{2}}$ and $Y_{\lambda}$ are only well defined modulo measures supported on the light cone in Fourier space. Because of this, it will be convenient for us to include an extra $L^{\infty}\left(L^{2}\right)$ norm in the definition of our function spaces. This represents the inclusion in the above norms of solutions to the wave equation with $L^{2}$ initial data. Adding everything together, we are led to define the following fixed frequency (semi) norms:

$$
\|u\|_{F_{\lambda}}=\left(X_{\lambda, 1}^{\frac{1}{2}}+Y_{\lambda}\right) \cap S_{\lambda}\left(L^{\infty}\left(L^{2}\right)\right) .
$$

Unfortunately, the above norm is still not strong enough for us to be able to iterate equations of the form (1) which contain derivatives. This is due to a very specific Low $\times$ High frequency interaction in quadratic non-linearities. Fortunately, this problem has been effectively handled by Tataru in [15, based on ideas from 7 ] and 8 . What is necessary is to add some extra $L^{1}\left(L^{\infty}\right)$ norms on "outer block" 
regions of Fourier space. This is the essence of the norm (20) above, which is a slight variant of that which appeared in [15]. This leads to our second main dyadic norm:

$$
\|u\|_{G_{\lambda}}=\left(X_{\lambda, 1}^{\frac{1}{2}}+Y_{\lambda}\right) \cap S_{\lambda}\left(L^{\infty}\left(L^{2}\right)\right) \cap Z_{\lambda} .
$$

Finally, the spaces we will iterate in are produced by adding the appropriate number of derivatives combined with the necessary Besov structures:

$$
\begin{aligned}
\|u\|_{F^{s}}^{2} & =\sum_{\lambda} \lambda^{2 s}\|u\|_{F_{\lambda}}^{2}, \\
\|u\|_{G^{s}} & =\sum_{\lambda} \lambda^{s}\|u\|_{G_{\lambda}} .
\end{aligned}
$$

Due to the need for precise microlocal decompositions, of crucial importance to us will be the boundedness of certain multipliers on the components (18)-(19) of our function spaces as well as mixed Lebesgue spaces. We state these as follows:

Lemma 3.1 (Multiplier boundedness).

(1) The following multipliers are given by $L^{1}$ kernels: $\lambda^{-1} \nabla S_{\lambda}, S_{\lambda, d}^{\omega}, S_{\lambda, \bullet \leqslant d}^{\omega}$, and $(\lambda d) \Xi^{-1} S_{\lambda, d}^{\omega}$. In particular, all of these are bounded on every mixed Lebesgue space $L^{q}\left(L^{r}\right)$.

(2) The following multipliers are bounded on the spaces $L^{q}\left(L^{2}\right)$, for $1 \leqslant q \leqslant \infty$ : $S_{\lambda, d}$ and $S_{\lambda, \bullet \leqslant d}$.

Proof of Lemma 3.1 (1). First, notice that after a rescaling, the symbol for the multiplier $\lambda^{-1} \nabla S_{\lambda}$ is a $C^{\infty}$ bump function with $O(1)$ support. Thus, its kernel is in $L^{1}$ with norm independent of $\lambda$.

For the remainder of the operators listed in (1) above, it suffices to work with $(\lambda d) \Xi^{-1} S_{\lambda, d}^{\omega}$. The boundedness of the others follows from a similar argument. We let $\chi^{ \pm}$denote the symbol of this operator cut off in the upper resp. lower half plane. After a rotation in the spatial domain, we may assume that the spatial projection of $\chi^{ \pm}$is directed along the positive $\xi_{1}$ axis. Now look at $\chi^{+}(s, \eta)$ with coordinates:

$$
\begin{aligned}
s & =\frac{1}{\sqrt{2}}\left(\tau-\xi_{1}\right), \\
\eta_{1} & =\frac{1}{\sqrt{2}}\left(\tau+\xi_{1}\right), \\
\eta^{\prime} & =\xi^{\prime} .
\end{aligned}
$$

It is apparent that $\chi^{+}(s, \eta)$ has support in a box of dimension $\sim \lambda \times \sqrt{\lambda d} \times \ldots \times$ $\sqrt{\lambda d} \times d$ with sides parallel to the coordinate axis and longest side in the $\eta_{1}$ direction and shortest side in the $s$ direction. Furthermore, a direction calculation shows that one has the bounds:

$$
\left|\partial_{\eta_{1}}^{N} \chi^{+}\right| \leqslant C_{N} \lambda^{-N}, \quad\left|\partial_{\eta^{\prime}}^{N} \chi^{+}\right| \leqslant C_{N}(\lambda d)^{-N / 2}, \quad\left|\partial_{s}^{N} \chi^{+}\right| \leqslant C_{N} d^{-N} .
$$


Therefore, we have that $\chi^{+}$yields an $L^{1}$ kernel. A similar argument works for the cutoff function $\chi^{-}$, using the rotation:

$$
\begin{aligned}
s & =\frac{1}{\sqrt{2}}\left(\tau+\xi_{1}\right), \\
\eta_{1} & =\frac{1}{\sqrt{2}}\left(-\tau+\xi_{1}\right), \\
\eta^{\prime} & =\xi^{\prime}
\end{aligned}
$$

Proof of Lemma 3.1 (2). We will argue here for $S_{\lambda, d}$. The estimates for the others follow similarly. If we denote by $K^{ \pm}(t, x)$ the convolution kernel associated with $S_{\lambda, d}^{ \pm}$, then a simple calculation shows that:

$$
e^{\mp 2 \pi i t|\xi|} \widehat{K^{ \pm}}(t, \xi)=\int e^{2 \pi i t \tau} \psi(\tau, \xi) d \tau,
$$

where $\operatorname{supp}\{\psi\}$ is contained in a box of dimension $\sim \lambda \times \ldots \times \lambda \times d$ with sides along the coordinate axis and short side in the $\tau$ direction. Furthermore, one has the estimate:

$$
\left|\partial_{\tau}^{N} \psi\right| \leqslant C_{N} d^{-N} .
$$

This shows that we have the bound:

$$
\left\|\widehat{K^{ \pm}}\right\|_{L_{\tau}^{1}\left(L_{\xi}^{\infty}\right)} \lesssim 1
$$

independent of $\lambda$ and $d$. Thus, we get the desired bounds for the convolution kernels.

As an immediate application of the above lemma, we show that the extra $Z_{\lambda}$ intersection in the $G_{\lambda}$ norm above only effects the $X_{\lambda, 1}^{\frac{1}{2}}$ portion of things.

Lemma 3.2 (Outer block estimate on $Y_{\lambda}$ ). For $5<n$, one has the following uniform inclusion:

$$
Y_{\lambda} \subseteq Z_{\lambda}
$$

proof of (25). It is enough to show that:

$$
\left(\sum_{\omega}\left\|\Xi^{-1} S_{\lambda, d}^{\omega} u\right\|_{L^{1}\left(L^{\infty}\right)}^{2}\right)^{\frac{1}{2}} \lesssim \lambda^{\frac{n-4}{2}}\left(\frac{d}{\lambda}\right)^{\frac{n-5}{4}}\left\|S_{\lambda} u\right\|_{L^{1}\left(L^{2}\right)} .
$$

First, using a local Sobolev embedding, we see that:

$$
\left\|B_{\lambda,(\lambda d)^{\frac{1}{2}}}^{\omega} \Xi^{-1} S_{\lambda, d}^{\omega} u\right\|_{L^{1}\left(L^{\infty}\right)} \lesssim \lambda^{\frac{n+1}{4}} d^{\frac{n-1}{4}}\left\|\Xi^{-1} S_{\lambda, d}^{\omega} u\right\|_{L^{1}\left(L^{2}\right)} .
$$


Therefore, using the boundedness Lemma 3.1 it suffices to note that by Minkowski's inequality we can bound:

$$
\begin{aligned}
\left(\sum_{\omega}\left(\int\left\|S_{\lambda, d}^{\omega} u\right\|_{L_{x}^{2}}\right)^{2}\right)^{\frac{1}{2}} & \lesssim \int\left(\sum_{\omega}\left\|S_{\lambda, d}^{\omega} u\right\|_{L_{x}^{2}}^{2}\right)^{\frac{1}{2}} \\
& \lesssim\left\|S_{\lambda, d} u\right\|_{L^{1}\left(L^{2}\right)}
\end{aligned}
$$

The last line of the above proof showed that it is possible to bound a square sum over an angular decomposition of a given function in $L^{1}\left(L^{2}\right)$. It is also clear that this same procedure works for the $X_{\lambda, 1}^{\frac{1}{2}}$ spaces because one can use Minkowski's inequality for the $\ell^{1}$ sum with respect to the cone variable $d$. This fact will be of great importance in what follows and we record it here as:

Lemma 3.3 (Angular reconstruction of norms). Given a test function $u$ and parameter $\delta \leqslant \lambda$, one can bound:

$$
\left(\sum_{\omega}\left\|B_{\lambda, \delta}^{\omega} u\right\|_{X_{\lambda, 1}^{\frac{1}{2}}, Y_{\lambda}}^{2}\right)^{\frac{1}{2}} \lesssim\|u\|_{X_{\lambda, 1}^{\frac{1}{2}}, Y_{\lambda}} .
$$

\section{Structure of the $F_{\lambda}$ SPaces}

The purpose of this section is to clarify some remarks of the previous section and write down two integral formulas for functions in the $F_{\lambda}$ space. This material is all more or less standard in the literature and we include it here primarily because the notation will be useful for our scattering result. Our first order of business is to write down a decomposition for functions in the $F_{\lambda}$ space:

Lemma 4.1 ( $F_{\lambda}$ decomposition). For any $u_{\lambda} \in F_{\lambda}$, one can write:

$$
u_{\lambda}=u_{\dot{X}_{\lambda}}+u_{X_{\lambda, 1}^{1 / 2}}+u_{Y_{\lambda}}
$$

where $u_{X_{\lambda}}$ is a solution to the homogeneous wave equation, $u_{X_{\lambda, 1}^{1 / 2}}$ is the Fourier transform of an $L^{1}$ function, and $u_{Y_{\lambda}}$ satisfies:

$$
u_{Y_{\lambda}}(0)=\partial_{t} u_{Y_{\lambda}}(0)=0
$$

Furthermore, one has the norm bounds:

$$
\frac{1}{C}\left\|u_{\lambda}\right\|_{F_{\lambda}} \leqslant\left(\left\|u_{\dot{X}_{\lambda}}\right\|_{L^{\infty}\left(L^{2}\right)}+\left\|u_{X_{\lambda, 1}^{1 / 2}}\right\|_{X_{\lambda, 1}^{\frac{1}{2}}}+\left\|u_{Y_{\lambda}}\right\|_{Y_{\lambda}}\right) \leqslant C\left\|u_{\lambda}\right\|_{F_{\lambda}} .
$$

We now show that the two inhomogeneous terms on the right hand side of (27) can be written as integrals over solutions to the wave equation with $L^{2}$ data. This fact will be of crucial importance to us in the sequel. The first formula is simply a restatement of (12): 
Lemma 4.2 (Duhamel's principle). Using the same notation as above, for any $u_{Y_{\lambda}}$, one can write:

$$
u_{Y_{\lambda}}(t)=-\int_{0}^{t}\left|D_{x}\right|^{-1} \sin \left((t-s)\left|D_{x}\right|\right) \square u_{Y_{\lambda}}(s) d s .
$$

Likewise, one can write the $u_{X_{\lambda, 1}^{1 / 2}}$ portion of the sum (27) as an integral over modulated solutions to the wave equation be foliating Fourier space by forward and backward facing light-cones:

Lemma 4.3 ( $X_{\lambda, 1}^{\frac{1}{2}}$ Trace lemma). For any $u_{X_{\lambda, 1}^{1 / 2}}$, let $u_{X_{\lambda, 1}^{1 / 2}}^{ \pm}$denote its restriction to the frequency half space $0< \pm \tau$. Then one can write:

$$
u_{X_{\lambda, 1}^{1 / 2}}^{ \pm}(t)=\int e^{2 \pi i t\left(s \pm\left|D_{x}\right|\right)} u_{\lambda, s}^{ \pm} d s
$$

where $u_{\lambda, s}^{ \pm}$is the spatial Fourier transform of $\widetilde{u^{ \pm}}$restricted to the $s^{\text {th }}$ translate of the forward or backward light-cone light cone in Fourier space, i.e.:

$$
{\widehat{u^{ \pm}}}_{\lambda, s}(\xi)=\int \delta(\tau-s \mp|\xi|) \widetilde{u^{ \pm}}(\tau, \xi) d \tau \text {. }
$$

In particular. one has the formula:

$$
\int\left\|u_{\lambda, s}^{ \pm}\right\|_{L^{2}} d s \lesssim\left\|u_{X_{\lambda, 1}^{1 / 2}}^{ \pm}\right\|_{X_{\lambda, 1}^{\frac{1}{2}}}
$$

\section{Strichartz estimates}

Our inductive estimates will be based on a method of bilinear decompositions and local Strichartz estimates as in the work [15]. We first state the standard Strichartz from which the local estimates follow.

Lemma 5.1 (Homogeneous Strichartz estimates (see [3])). Let $5<n, \sigma=\frac{n-1}{2}$, and suppose $u$ is a given function of the spatial variable only. Then if $\frac{1}{q}+\frac{\sigma}{r} \leqslant \frac{\sigma}{2}$ and $\frac{1}{q}+\frac{n}{r}=\frac{n}{2}-\gamma$, the following estimate holds:

$$
\left\|e^{ \pm 2 \pi i t\left|D_{x}\right|} P_{\bullet \leqslant \lambda} u\right\|_{L_{t}^{q}\left(L_{x}^{r}\right)} \lesssim \lambda^{\gamma}\left\|P_{\bullet} \leqslant \lambda u\right\|_{L^{2}} .
$$

Combining the $L^{2}\left(L^{\frac{2(n-1)}{n-3}}\right)$ endpoint of the above estimate with a local Sobolev in the spatial domain, we arrive at the following local version of (32):

Lemma 5.2 (Local Strichartz estimate). Let $5<n$, then the following estimate holds:

$$
\left\|e^{ \pm 2 \pi i t\left|D_{x}\right|} B_{\lambda,(\lambda d)^{\frac{1}{2}}}^{\omega} u\right\|_{L_{t}^{2}\left(L_{x}^{\infty}\right)} \lesssim \lambda^{\frac{n+1}{4}} d^{\frac{n-3}{4}}\left\|B_{\lambda,(\lambda d)^{\frac{1}{2}}}^{\omega} u\right\|_{L^{2}}
$$


Using the integral formulas (29) and (30), we can transfer the above estimates to the $F_{\lambda}$ spaces:

Lemma 5.3 ( $F_{\lambda}$ Strichartz estimates). Let $5<n$ and set $\sigma=\frac{n-1}{2}$. Then if $\frac{1}{q}+\frac{\sigma}{r} \leqslant \frac{\sigma}{2}$ and $\frac{1}{q}+\frac{n}{r}=\frac{n}{2}-\gamma$, the following estimates hold:

$$
\begin{gathered}
\left\|S_{\lambda} u\right\|_{L^{q}\left(L^{r}\right)} \\
\left(\sum_{\omega}\left\|S_{\lambda, \bullet \leqslant d}^{\alpha} u\right\|_{L^{2}\left(L^{\infty}\right)}^{2}\right)^{\frac{1}{2}} \\
\lesssim \lambda_{F_{\lambda}},
\end{gathered}
$$

Proof of Lemma 5.3. It suffices to prove the estimate (34), as the estimate (35) follows from this and a local Sobolev embedding combined with the resumming formula (26). Using the decomposition (4.1) and the angular reconstruction formula (26), it is enough to prove (34) for functions $u_{X_{\lambda, 1}^{1 / 2}}$ and $u_{Y_{\lambda}}$. Using the integral formula (30), we see immediately that:

$$
\begin{aligned}
\left\|u_{X_{\lambda, 1}^{1 / 2}}\right\|_{L^{q}\left(L^{r}\right)} & \leqslant \sum_{ \pm} \int\left\|e^{ \pm 2 \pi i t\left|D_{x}\right|} u_{\lambda, s}^{ \pm}\right\|_{L^{q}\left(L^{r}\right)} d s \\
& \lesssim \lambda^{\gamma} \sum_{ \pm} \int\left\|u_{\lambda, s}^{ \pm}\right\|_{L^{2}} d s \\
& \lesssim \lambda^{\gamma}\left\|u_{X_{\lambda, 1}^{1 / 2}}\right\|_{X_{\lambda, 1}^{\frac{1}{2}}} .
\end{aligned}
$$

For the $u_{Y_{\lambda}}$ portion of things, we can chop the function up into a fixed number of space-time angular sectors using $L^{1}$ convolution kernels. Doing this and using $R_{\alpha}$ to denote an operator from the set $\left\{I, \partial_{i}\left|D_{x}\right|^{-1}\right\}$, we estimate:

$$
\begin{aligned}
\left\|u_{Y_{\lambda}}\right\|_{L^{q}\left(L^{r}\right)} & \leqslant \lambda^{-1} \sum_{\alpha}\left\|\partial_{\alpha} u_{Y_{\lambda}}\right\|_{L^{q}\left(L^{r}\right)} \\
& \lesssim \lambda^{-1} \sum_{ \pm, \alpha} \int\left\|e^{ \pm 2 \pi i t\left|D_{x}\right|}\left(e^{\mp 2 \pi i s\left|D_{x}\right|} R_{\alpha} \square u_{Y_{\lambda}}(s, x)\right)\right\|_{L_{t}^{q}\left(L_{x}^{r}\right)} d s \\
& \lesssim \lambda^{\gamma} \lambda^{-1} \sum_{\alpha} \int\left\|e^{\mp 2 \pi i s\left|D_{x}\right|} R_{\alpha} \square u_{Y_{\lambda}}(s, x)\right\|_{L_{x}^{2}} d s \\
& \leqslant \lambda^{\gamma}\left\|u_{Y_{\lambda}}\right\|_{Y_{\lambda}} .
\end{aligned}
$$

A consequence of (34) is that we have the embedding:

$$
X_{\lambda, 1}^{\frac{1}{2}} \subseteq L^{\infty}\left(L^{2}\right) .
$$

Using a simple approximation argument along with uniform convergence, we arrive at the following energy estimate for the $F^{s}$ and $G^{s}$ spaces: 
Lemma 5.4 (Energy estimates). For space-time functions $u$, one has the following estimates:

$$
\begin{aligned}
\|u\|_{C\left(\dot{H}^{s}\right) \cap C^{(1)}\left(\dot{H}^{s-1}\right)} & \lesssim\|u\|_{F^{s}}, \\
\|u\|_{C\left(\dot{B}^{s}\right) \cap C^{(1)}\left(\dot{B}^{s-1}\right)} & \lesssim\|u\|_{G^{s}} .
\end{aligned}
$$

Also, by duality and the estimate (36), we have that:

$$
\lambda \Xi^{-1} L^{1}\left(L^{2}\right) \subseteq \lambda \Xi^{-1} X_{\lambda, \infty}^{-\frac{1}{2}} \subseteq X_{\lambda, \infty}^{\frac{1}{2}} .
$$

This proves shows:

Lemma 5.5 ( $L^{2}$ estimate for $\left.Y_{\lambda}\right)$. The following inclusion holds uniformly:

$$
d^{\frac{1}{2}} S_{\lambda, d}\left(Y_{\lambda}\right) \subseteq L^{2}\left(L^{2}\right)
$$

in particular, by dyadic summing one has:

$$
d^{\frac{1}{2}} S_{\lambda, d \leqslant \bullet}\left(F_{\lambda}\right) \subseteq L^{2}\left(L^{2}\right) .
$$

\section{SCATTERING}

It turns out that our scattering result, Theorem 1.2 is implicitly contained in the function spaces $F^{s}$ and $G^{s}$. That is, there is scattering in these spaces independently of any specific equation being considered. Therefore, to prove Theorem 1.2 it will only be necessary to show that our solution to (10) belongs to these spaces.

Using a simple approximation argument, it suffices to deal with things at fixed frequency. Because the estimates in Theorem 1.2 deal with more than one derivative, we will show that:

Lemma 6.1 ( $F_{\lambda}$ scattering). For any function $u_{\lambda} \in F_{\lambda}$, there exists a set of initial data $\left(f_{\lambda}^{ \pm}, g_{\lambda}^{ \pm}\right) \in P_{\lambda}\left(L^{2}\right) \times \lambda P_{\lambda}\left(L^{2}\right)$ such that the following asymptotic holds:

$$
\begin{aligned}
\lim _{t \rightarrow \infty}\left\|u_{\lambda}(t)-W\left(f_{\lambda}^{+}, g_{\lambda}^{+}\right)(t)\right\|_{\dot{H}^{1} \cap \partial_{t}\left(L^{2}\right)} & =0, \\
\lim _{t \rightarrow-\infty}\left\|u_{\lambda}(t)-W\left(f_{\lambda}^{-}, g_{\lambda}^{-}\right)(t)\right\|_{\dot{H}^{1} \cap \partial_{t}\left(L^{2}\right)} & =0 .
\end{aligned}
$$

Proof of Lemma 6.1. Using the notation of Section 4 we may write:

$$
u_{\lambda}=u_{\dot{X}_{\lambda}}+u_{X_{\lambda, 1}^{1 / 2}}^{+}+u_{X_{\lambda, 1}^{1 / 2}}^{-}+u_{Y_{\lambda}},
$$

We now define the scattering data implicitly by the relations:

$$
\begin{aligned}
& W\left(f_{\lambda}^{+}, g_{\lambda}^{+}\right)(t)=u_{\dot{X}_{\lambda}}-\int_{0}^{\infty}\left|D_{x}\right|^{-1} \sin \left(\left|D_{x}\right|(t-s)\right) \square u_{Y_{\lambda}}(s) d s, \\
& W\left(f_{\lambda}^{-}, g_{\lambda}^{-}\right)(t)=u_{\dot{X}_{\lambda}}-\int_{-\infty}^{0}\left|D_{x}\right|^{-1} \sin \left(\left|D_{x}\right|(t-s)\right) \square u_{Y_{\lambda}}(s) d s .
\end{aligned}
$$


Using the fact that $\square u_{Y_{\lambda}}$ has finite $L^{1}\left(L^{2}\right)$ norm, it suffices to show that one has the limits:

$$
\lim _{t \rightarrow \pm \infty}\left\|u_{X_{\lambda, 1}^{1 / 2}}^{+}(t)+u_{X_{\lambda, 1}^{1 / 2}}^{-}(t)\right\|_{\dot{H}^{1} \cap \partial_{t}\left(L^{2}\right)}=0
$$

Squaring this, we see that we must show the limits:

$$
\begin{aligned}
& \lim _{t \rightarrow \pm \infty} \int\left|D_{x}\right| u_{X_{\lambda, 1}^{1 / 2}}^{+}(t) \overline{\left|D_{x}\right| u_{X_{\lambda, 1}^{1 / 2}}^{ \pm}}(t)=0, \\
& \lim _{t \rightarrow \pm \infty} \int \partial_{t} u_{X_{\lambda, 1}^{1 / 2}}^{+}(t) \overline{\partial_{t} u_{X_{\lambda, 1}^{ \pm}}^{ \pm}}(t)=0 .
\end{aligned}
$$

We'll only deal here with the limit (42), as the limit (43) follows from a virtually identical argument. Using the trace formula (30) along with the Plancherel theorem, we compute:

(L.H.S.) (42)

$$
=\lim _{t \rightarrow \pm \infty} \int e^{2 \pi i t(|\xi| \mp|\xi|)}|\xi|^{2} \int e^{2 \pi i t s_{1}} \widehat{u_{\lambda, s_{1}+s_{2}}^{+}}(\xi) \overline{\widehat{u_{\lambda, s_{2}}^{ \pm}}(\xi)} d s_{1} d s_{2} d \xi .
$$

By (31) we have the bounds:

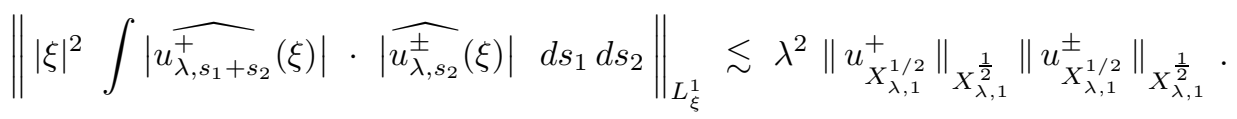

This shows that the function:

$$
H_{t}(\xi)=|\xi|^{2} \int e^{2 \pi i t s_{1}} \widehat{u_{\lambda, s_{1}+s_{2}}^{+}}(\xi) \widehat{\widehat{u_{\lambda, s_{2}}^{ \pm}}(\xi)} d s_{1} d s_{2}
$$

is bounded pointwise by an $L^{1}$ function uniformly in $t$. Therefore, by the dominated convergence theorem, it suffices to show that we in fact have that $\lim _{t \rightarrow \pm \infty} H_{t}=0$. To see this, notice that by the above bounds in conjunction with Fubini's theorem, we have that the integral:

$$
|\xi|^{2} \int \widehat{u_{\lambda, s_{1}+s_{2}}^{+}}(\xi) \overline{\widehat{u_{\lambda, s_{2}}^{ \pm}}(\xi)} d s_{2}
$$

is in $L_{s_{1}}^{1}$. The result now follows from the Riemann Lebesgue Lemma. Explicitly, one has that for almost every fixed $\xi$, the following limit holds:

$$
\lim _{t \rightarrow \pm \infty} H_{t}(\xi)=\lim _{t \rightarrow \pm \infty}|\xi|^{2} \int e^{2 \pi i t s_{1}} \widehat{u_{\lambda, s_{1}+s_{2}}^{+}}(\xi) \overline{\widehat{u_{\lambda, s_{2}}^{ \pm}}(\xi)} d s_{1} d s_{2}=0
$$

\section{Inductive Estimates I}

Our solution to (1) will be produced through the usual procedure of Picard iteration. Because the initial data and our function spaces are both invariant with respect to the scaling (3), any iteration procedure must effectively be global in time. Therefore, we shall have no need of an auxiliary time cutoff system as in the works [6-15. Instead, we write (1) directly as an integral equation:

$$
\phi=W(f, g)+\square^{-1} \mathcal{N}(\phi, D \phi) .
$$


By the contraction mapping principle and the quadratic nature of the nonlinearity, to produce a solution to (44) which satisfies the regularity assumptions of our main theorem, it suffices to prove the following two sets of estimates:

Theorem 7.1 (Solution of the division problem). Let $5<n$, then the $F$ and $G$ spaces solve the division problem for quadratic wave equations in the sense that for any of the model systems we have written above: YM, WM, or MD, one has the following estimates:

$$
\begin{aligned}
\left\|\square^{-1} \mathcal{N}(u, D v)\right\|_{G^{s_{c}}} & \lesssim\|u\|_{G^{s_{c}}}\|v\|_{G^{s_{c}}}, \\
\left\|\square^{-1} \mathcal{N}(u, D v)\right\|_{F^{s}} & \lesssim\|u\|_{G^{s_{c}}}\|v\|_{F^{s}}+\|u\|_{F^{s}}\|v\|_{G^{s_{c}}} .
\end{aligned}
$$

The remainder of the paper is devoted to the proof of Theorem [7.1 In what follows, we will work exclusively with the equation:

$$
\phi=W(f, g)+\square^{-1}(\phi \nabla \phi) .
$$

In this case, we set $s_{c}=\frac{n-2}{2}$. The proof of Theorem 7.1] for the other model equations can be achieved through a straightforward adaptation of the estimates we give here. In fact, after the various derivatives and values of $s_{c}$ are taken into account, the proof in these cases follows verbatim from estimates (49) and (50) below.

Our first step is to take a Littlewood-Paley decomposition of $\square^{-1}(u \nabla v)$ with respect to space-time frequencies:

$$
\square^{-1}(u \nabla v)=\sum_{\mu_{i}} \square^{-1}\left(S_{\mu_{1}} u \nabla S_{\mu_{2}} v\right) .
$$

We now follow the standard procedure of splitting the sum (48) into three pieces depending on the cases $\mu_{1} \ll \mu_{2}, \mu_{2} \ll \mu_{1}$, and $\mu_{2} \sim \mu_{1}$. Therefore, due to the $\ell^{1}$ Besov structure in the $F$ spaces, in order to prove both (45) and (46), it suffices to show the two estimates:

$$
\begin{gathered}
\left\|\square^{-1}\left(S_{\mu_{1}} u \nabla S_{\mu_{2}} v\right)\right\|_{G_{\lambda}} \lesssim \lambda^{-1} \mu_{1}^{\frac{n}{2}}\|u\|_{F_{\mu_{1}}}\|v\|_{F_{\mu_{2}}}, \mu_{1} \sim \mu_{2}, \\
\left\|\square^{-1}\left(S_{\mu} u \nabla S_{\lambda} v\right)\right\|_{G_{\lambda}} \lesssim \mu^{\frac{n-2}{2}}\|u\|_{G_{\mu}}\|v\|_{F_{\lambda}}, \mu \ll \lambda .
\end{gathered}
$$

Notice that after some weight trading, the estimates (45) and (46) follow from (50) in the case where $\mu_{2} \ll \mu_{1}$.

proof of (49). It is enough if we show the following two estimates:

$$
\begin{aligned}
\left\|S_{\lambda}\left(S_{\mu_{1}} u \nabla S_{\mu_{2}} v\right)\right\|_{L^{1}\left(L^{2}\right)} & \lesssim \mu_{1}^{\frac{n}{2}}\|u\|_{F_{\mu_{1}}}\|v\|_{F_{\mu_{2}}}, \mu_{1} \sim \mu_{2}, \\
\left\|S_{\lambda} \square^{-1}\left(S_{\mu_{1}} u \nabla S_{\mu_{2}} v\right)\right\|_{L^{\infty}\left(L^{2}\right)} & \lesssim \lambda^{-1} \mu_{1}^{\frac{n}{2}}\|u\|_{F_{\mu_{1}}}\|v\|_{F_{\mu_{2}}}, \mu_{1} \sim \mu_{2} .
\end{aligned}
$$


In fact, it suffices to prove (51). To see this, notice that one has the formula:

$$
\left[S_{\lambda}, \square^{-1}\right] G=W\left(E * S_{\lambda} G\right)-S_{\lambda} W(E * G) .
$$

Thus, after multiplying by $S_{\lambda}$, we see that:

$$
\begin{aligned}
S_{\lambda}\left[S_{\lambda}, \square^{-1}\right] G & =P_{\lambda}\left(W\left(E * S_{\lambda} G\right)-S_{\lambda} W(E * G)\right), \\
& =W\left(E * S_{\lambda} P_{\lambda} G\right)-S_{\lambda} W\left(E * P_{\lambda} G\right), \\
& =S_{\lambda}\left[S_{\lambda}, \square^{-1}\right] P_{\lambda} G .
\end{aligned}
$$

Therefore, by the (approximate) idempotence of $S_{\lambda}$ one has:

$$
\begin{aligned}
S_{\lambda} \square^{-1} G & =S_{\lambda} \square^{-1} S_{\lambda} G+S_{\lambda}\left[S_{\lambda}, \square^{-1}\right] G, \\
& =S_{\lambda} \square^{-1} S_{\lambda} G+S_{\lambda}\left[S_{\lambda}, \square^{-1}\right] P_{\lambda} G .
\end{aligned}
$$

Thus, by the boundedness of $S_{\lambda}$ on the spaces $L^{\infty}\left(L^{2}\right)$ the energy estimate, one can bound:

$$
\left\|S_{\lambda} \square^{-1} G\right\|_{L^{\infty}\left(L^{2}\right)} \lesssim \lambda^{-1}\left(\left\|S_{\lambda} G\right\|_{L^{1}\left(L^{2}\right)}+\left\|P_{\lambda} G\right\|_{L^{1}\left(L^{2}\right)}+\left\|S_{\lambda} P_{\lambda} G\right\|_{L^{1}\left(L^{2}\right)}\right) .
$$

We now use the fact that the multipliers $S_{\lambda}$ and $P_{\lambda}$ are both bounded on the space $L^{1}\left(L^{2}\right)$ to reduce things to the estimate:

$$
\begin{aligned}
\left\|S_{\mu_{1}} u \nabla S_{\mu_{2}} v\right\|_{L^{1}\left(L^{2}\right)} \lesssim & \left\|S_{\mu_{1}} u\right\|_{L^{2}\left(L^{4}\right)}\left\|\nabla S_{\mu_{2}} v\right\|_{L^{2}\left(L^{4}\right)}, \\
& \mu_{1}^{\frac{n-2}{4}} \mu_{2}^{\frac{n+2}{4}}\|u\|_{F_{\mu_{1}}}\|v\|_{F_{\mu_{2}} .} .
\end{aligned}
$$

Taking into account the the bound $\mu_{1} \sim \mu_{2}$, the claim now follows.

Next, we'll deal with the estimate (150). For the remainder of the paper we shall fix both $\lambda$ and $\mu$ and assume they such that $\mu \ll \lambda$ for a fixed constant. We now decompose the product $S_{\lambda}\left(S_{\mu} u \nabla S_{\lambda} v\right)$ into a sum of three pieces:

$$
S_{\lambda}\left(S_{\mu} u \nabla S_{\lambda} v\right)=A+B+C,
$$

where

$$
\begin{aligned}
& A=S_{\lambda}\left(S_{\mu} u \nabla S_{\lambda, c \mu \leqslant \bullet} v\right), \\
& B=S_{\lambda, c \mu \leqslant \bullet}\left(S_{\mu} u \nabla S_{\lambda, \bullet<c \mu} v\right), \\
& C=S_{\lambda, \bullet<c \mu}\left(S_{\mu} u \nabla S_{\lambda, \bullet<c \mu} v\right) .
\end{aligned}
$$

Here $c$ is a suitably small constant which will be chosen later. It will be needed to make explicit a dependency between some of the constants which arise in a specific frequency localization in the sequel. We now work to recover the estimate (50) for each of the three above terms separately. 
proof of (50) for the term A. Following the remarks at the beginning of the proof of (49), it suffices to compute:

$$
\begin{aligned}
\| S_{\lambda}\left(S_{\mu} u \nabla S_{\lambda, c \mu \leqslant \bullet} v \|_{L^{1}\left(L^{2}\right)}\right. & \lesssim \lambda\left\|S_{\mu} u\right\|_{L^{2}\left(L^{\infty}\right)}\left\|S_{\lambda, c \mu \leqslant \bullet} v\right\|_{L^{2}\left(L^{2}\right)}, \\
& \lesssim \lambda \mu^{\frac{n-1}{2}}\|u\|_{F_{\mu}}(c \mu)^{-\frac{1}{2}}\|v\|_{F_{\lambda}}, \\
& \lesssim c^{-1} \lambda \mu^{\frac{n-2}{2}}\|u\|_{F_{\mu}}\|v\|_{F_{\lambda}} .
\end{aligned}
$$

For a fixed $c$, we obtain the desired result.

We now move on to showing the inclusion (50) for the $B$ term above. In this range, we are forced to work outside the context of $L^{1}\left(L^{2}\right)$ estimates. This is the reason we have included the $L^{2}\left(L^{2}\right)$ based $X_{\lambda, 1}^{\frac{1}{2}}$ spaces. This also means that we will need to recover $Z_{\lambda}$ norms by hand (because they are only covered by the $Y_{\lambda}$ spaces). However, because this last task will require a somewhat finer analysis than what we will do in this section, we contend ourselves here with showing:

proof of the $X_{\lambda, 1}^{\frac{1}{2}} \cap S_{\lambda}\left(L^{\infty}\left(L^{2}\right)\right)$ estimates for the term $B$. Our first task will be deal with the energy estimate which we write as:

$$
\left\|S_{\lambda} \square^{-1} S_{\lambda, c \mu \leqslant \bullet}\left(S_{\mu} u \nabla S_{\lambda, \bullet<c \mu} v\right)\right\|_{L^{\infty}\left(L^{2}\right)} \lesssim \mu^{\frac{n-2}{2}}\|u\|_{F_{\mu}}\|v\|_{F_{\lambda}} .
$$

For $G$ supported away from the light-cone in Fourier space, we have the identity:

$$
S_{\lambda} \square^{-1} S_{\lambda} G=\Xi^{-1} S_{\lambda} G-W\left(\Xi^{-1} P_{\lambda} S_{\lambda} G\right) .
$$

Therefore, by using the energy estimate for the $X_{\lambda, 1}^{\frac{1}{2}}$ space, this allows us to estimate:

$$
\begin{aligned}
\left\|S_{\lambda} \square^{-1} S_{\lambda} G\right\|_{L^{\infty}\left(L^{2}\right)} & \lesssim\left\|\Xi^{-1} S_{\lambda} G\right\|_{L^{\infty}\left(L^{2}\right)}+\left\|W\left(\Xi^{-1} P_{\lambda} S_{\lambda} G\right)\right\|_{L^{\infty}\left(L^{2}\right)} \\
& \lesssim\left\|\Xi^{-1} S_{\lambda} G\right\|_{L^{\infty}\left(L^{2}\right)} \\
& \lesssim\left\|\Xi^{-1} S_{\lambda} G\right\|_{X_{\lambda, 1}^{\frac{1}{2}}} .
\end{aligned}
$$

Therefore, we are left with estimating the term $B$ in the $X_{\lambda, 1}^{\frac{1}{2}}$ space. For a fixed distance $d$ from the cone, we compute that:

$$
\begin{aligned}
\left\|\Xi^{-1} S_{\lambda, d} S_{\lambda, c \mu \leqslant \bullet}\left(S_{\mu} u \nabla S_{\lambda, \bullet<c \mu} v\right)\right\|_{L^{2}\left(L^{2}\right)} & \lesssim d^{-1}\left\|S_{\mu} u\right\|_{L^{2}\left(L^{\infty}\right)}\left\|S_{\lambda} v\right\|_{L^{\infty}\left(L^{2}\right)} \\
& \lesssim d^{-1} \mu^{\frac{n-2}{2}}\|v\|_{F_{\mu}}\|u\|_{F_{\lambda}} .
\end{aligned}
$$

Summing $d^{\frac{1}{2}}$ times this last expression over all $c \mu \leqslant d$ yields:

$$
\begin{aligned}
\sum_{c \mu \leqslant d} d^{\frac{1}{2}} \| \Xi^{-1} S_{\lambda, d} S_{\lambda, c \mu \leqslant \bullet}\left(S_{\mu} u \nabla S_{\lambda, \bullet<c \mu} v\right) & \|_{L^{2}\left(L^{2}\right)} \\
& \lesssim \sum_{c \mu \leqslant d}\left(\frac{\mu}{d}\right)^{\frac{1}{2}} \mu^{\frac{n-2}{2}}\|v\|_{F_{\mu}}\|u\|_{F_{\lambda}} .
\end{aligned}
$$

For a fixed $c$ we obtain the desired result. 


\section{INTERLUdE: SOME BILINEAR DECOMPOSITIONS}

To proceed further, it will be necessary for us to take a closer look at the expression:

$$
S_{\lambda, d}^{\omega}\left(S_{\mu} u \nabla S_{\lambda, \bullet<c \mu} v\right), \quad c \mu \leqslant d,
$$

as well as the sum:

$$
C=S_{\lambda, \bullet<c \mu}\left(S_{\mu} u \nabla S_{\lambda, \bullet<c \mu} v\right)=C_{I}+C_{I I}+C_{I I I}
$$

where

$$
\begin{aligned}
C_{I} & =\sum_{d<c \mu} S_{\lambda, d}\left(S_{\mu, \bullet \leqslant d} u \nabla S_{\lambda, \bullet \leqslant d} v\right), \\
C_{I I} & =\sum_{d<c \mu} S_{\lambda, \bullet<d}\left(S_{\mu, \bullet \leqslant d} u \nabla S_{\lambda, d} v\right), \\
C_{I I I} & =\sum_{d \leqslant \mu} S_{\lambda, \bullet \min \{c \mu, d\}}\left(S_{\mu, d} u \nabla S_{\lambda, \bullet<\min \{c \mu, d\}} v\right) .
\end{aligned}
$$

We'll begin with a decomposition of $C_{I}$ and $C_{I I}$. The $C_{I I I}$ term is basically the same but requires a slightly more delicate analysis. All of the decompositions we compute here will be for a fixed $d$. The full decomposition will then be given by summing over the relevant values of $d$. Because our decompositions will be with respect to Fourier supports, it suffices to look at the convolution product of the corresponding cutoff functions in Fourier space. In what follows, we'll only deal with the $C_{I}$ term. It will become apparent that the same idea works for $C_{I I}$. Therefore, without loss of generality, we shall decompose the product:

$$
s_{\lambda, d}^{+}\left(s_{\mu, \bullet \leqslant d}^{ \pm} * s_{\lambda, \bullet \leqslant d}^{+}\right) \text {. }
$$

To do this, we use the standard device of restricting the angle of interaction in the above product. It will be crucial for us to be able to make these restrictions based only on the spatial Fourier variables, because we will need to reconstruct our decompositions through square-summing. For $\left(\tau^{\prime}, \xi^{\prime}\right) \in \operatorname{supp}\left\{s_{\mu, \bullet \leqslant d}^{ \pm}\right\}$and $(\tau, \xi) \in \operatorname{supp}\left\{s_{\lambda, \bullet \leqslant d}^{+}\right\} \quad$ we compute that:

$$
\begin{aligned}
O(d) & =|| \tau^{\prime}+\tau|-| \xi^{\prime}+\xi||, \\
& =|| \pm\left|\xi^{\prime}\right|+|\xi|+O(d)|-| \xi^{\prime}+\xi||, \\
& =|O(d)+| \pm\left|\xi^{\prime}\right|+|\xi||-| \xi^{\prime}+\xi|| .
\end{aligned}
$$

Using now the fact that $d<c \mu$ and $\mu<c \lambda$ to conclude that $\left|\xi^{\prime}\right| \sim \mu$ and $|\xi| \sim \lambda$, we see that one has the angular restriction:

$$
\mu \Theta_{ \pm \xi^{\prime}, \xi}^{2} \lesssim| \pm| \xi^{\prime}|+| \xi|-| \xi^{\prime}+\xi||=O(d) .
$$


In particular we have that $\Theta_{ \pm \xi^{\prime}, \xi} \lesssim \sqrt{\frac{d}{\mu}}$. This allows us to decompose the product (54) into a sum over angular regions with $O\left(\sqrt{\frac{d}{\mu}}\right)$ spread. The result is:

Lemma 8.1 (Wide angle decomposition). In the ranges stated for the $C_{I}$ term above, one can write:

$$
s_{\lambda, d}^{+}\left(s_{\mu, \bullet \leqslant d}^{ \pm} * s_{\lambda, \bullet \leqslant d}^{+}\right)=\sum_{\substack{\omega_{1}, \omega_{2}, \omega_{3}: \\\left|\omega_{1} \mp \omega_{2}\right| \sim(d / \mu)^{\frac{1}{2}} \\\left|\omega_{1}-\omega_{3}\right| \sim(d / \mu)^{\frac{1}{2}}}} b_{\lambda, \lambda\left(\frac{d}{\mu}\right)^{\frac{1}{2}}}^{\omega_{1}} s_{\lambda, d}^{+}\left(s_{\mu, \bullet \leqslant d}^{\omega_{2}} \pm b_{\lambda, \lambda\left(\frac{d}{\mu}\right)^{\frac{1}{2}}}^{\omega_{3}} s_{\lambda, \bullet \leqslant d}^{+}\right) .
$$

for the convolution of the associated cutoff functions in Fourier space.

We note here that the key feature in the decomposition (55) is that the sum is (essentially) diagonal in all three angles which appear there $\left(\omega_{1}, \omega_{2}, \omega_{3}\right)$. It is useful here to keep in mind the following diagram:
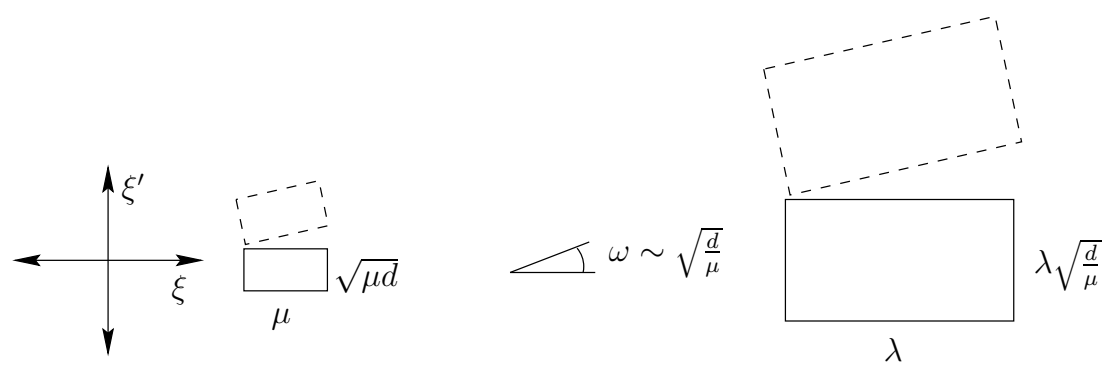

FIGURE 1. Spatial supports in the wide angle decomposition.

We now focus our attention on decomposing the convolution:

$$
s_{\lambda, \bullet \leqslant \min \{c \mu, d\}}^{+}\left(s_{\mu, d}^{ \pm} * s_{\lambda, \bullet \leqslant \min \{c \mu, d\}}^{+}\right) .
$$

If it is the case that $d \ll \mu$, then the same calculation which was used to produce (55) works and we end up with the same type of sum. However, if we are in the case where $d \sim \mu$, we need to compute things a bit more carefully in order to ensure that we may still decompose the multiplier $s_{\mu, d}^{ \pm}$using only restrictions in the spatial variable. To do this, we will now assume that things are set up so that $c \mu \ll d$. It is clear that all the previous decompositions can be made so that we can reduce things to this consideration. If we now take $\left(\tau^{\prime}, \xi^{\prime}\right) \in \operatorname{supp}\left\{s_{\mu, d}^{ \pm}\right\}$ and $(\tau, \xi) \in \operatorname{supp}\left\{s_{\lambda, \bullet \leqslant \min \{c \mu, d\}}^{+}\right\}$, we can use the facts that $\tau^{\prime}=O^{\mp}(d) \pm\left|\xi^{\prime}\right|$, $\tau=O(c \mu)+|\xi|$, and $|\xi| \gg \mu$ to compute that: 


$$
\begin{aligned}
O(c \mu) & =|| \tau^{\prime}+\tau|-| \xi^{\prime}+\xi||, \\
& =\left|O^{\mp}(d) \pm\right| \xi^{\prime}|+O(c \mu)+| \xi|-| \xi^{\prime}+\xi||,
\end{aligned}
$$

where the term $O^{\mp}(d)$ in the above expression is such that $\left|O^{\mp}(d)\right| \sim d$. In fact, one can see that the equality (57) forces $\pm O^{\mp}(d)<0$ on account of the fact that $\pm\left( \pm\left|\xi^{\prime}\right|+|\xi|-\left|\xi^{\prime}+\xi\right|\right)>0$ and the assumption $\left|O(c \mu)+O^{\mp}(d)\right| \sim d$. In particular, this means that we can multiply $s_{\mu, d}^{ \pm}$in the product (156) by the cutoff $s_{|\tau|<|\xi|}$ without effecting things. This in turn shows that we may decompose the product (56) based solely on restriction of the spatial Fourier variables, just as we did to get the sum in Lemma 8.1

We now return to the $C_{I}$ term. For the sequel, we will need to know what the contribution of the factor $S_{\lambda, \bullet \leqslant d} v$ to the following localized product is:

$$
S_{\lambda, d}^{\omega_{1}}\left(S_{\mu, \bullet \leqslant d} u \nabla S_{\lambda, \bullet \leqslant d} v\right) .
$$

Using Lemma 8.1 we see that we may write:

$$
s_{\lambda, d}^{\omega_{1}}\left(s_{\mu, \bullet \leqslant d} * s_{\lambda, \bullet \leqslant d}\right)=s_{\lambda, d}^{\omega_{1}}\left(s_{\mu, \bullet \leqslant d}^{\omega_{2}} * b_{\lambda, \lambda\left(\frac{d}{\mu}\right)^{\frac{1}{2}}}^{\omega_{3}} s_{\lambda, \bullet \leqslant d}\right),
$$

where $\left|\omega_{1}- \pm \omega_{2}\right| \sim\left|\omega_{3}- \pm \omega_{2}\right| \sim \sqrt{\frac{d}{\mu}}$. However, this can be refined significantly. To see this, assume that the spatial support of $s_{\lambda, d}^{\omega_{1}}$ lies along the positive $\xi_{1}$ axis. We'll label this block by $b_{\lambda,(\lambda d)^{\frac{1}{2}}}^{\omega_{1}}$. Because we are in the range where $\sqrt{\mu d} \ll \sqrt{\lambda d}$, we see that since for any $\xi \in \operatorname{supp}\left\{b_{\lambda, \lambda\left(\frac{d}{\mu}\right)^{\frac{1}{2}}}^{\omega_{3}}\right\}$ and $\xi^{\prime} \in \operatorname{supp}_{\xi^{\prime}}\left\{s_{\mu, \bullet \leqslant d}^{\omega_{2}}\right\}$ the sum $\xi+\xi^{\prime}$ must belong to $\operatorname{supp}\left\{b_{\lambda,(\lambda d)^{\frac{1}{2}}}^{\omega_{1}}\right\}$, we in fact have that $\xi$ itself must belong to a block of size $\lambda \times \sqrt{\lambda d} \times \ldots \times \sqrt{\lambda d}$. This allows us to write:

Lemma 8.2 (Small angle decomposition). In the ranges stated for the $C_{I}$ term above, we can write:

$$
s_{\lambda, d}^{\omega_{1}}\left(s_{\mu, \bullet \leqslant d} * s_{\lambda, \bullet \leqslant d}\right)=s_{\lambda, d}^{\omega_{1}}\left(s_{\mu, \bullet \leqslant d}^{\omega_{2}} * s_{\lambda, \bullet \leqslant d}^{\omega_{3}}\right) .
$$

where $\left|\omega_{1}-\omega_{3}\right| \sim \sqrt{\frac{d}{\lambda}}$, and $\left|\omega_{1}- \pm \omega_{2}\right| \sim \sqrt{\frac{d}{\mu}}$.

It is important to note here that if one were to sum the expression (59) over $\omega_{1}$, the resulting sum would be (essentially) diagonal in $\omega_{3}$, but there would be many $\omega_{1}$ which would contribute to a single $\omega_{2}$. This means that the resulting would not be diagonal in $\omega_{2}$ as was the case for the sum (55). It is helpful to visualize things through the following figure:

Our final task here is to mention an analog of Lemma 8.2 for the term (533). Here we can frequency localize the factor $S_{\lambda, \bullet<c \mu}$ in the product using the fact that one 

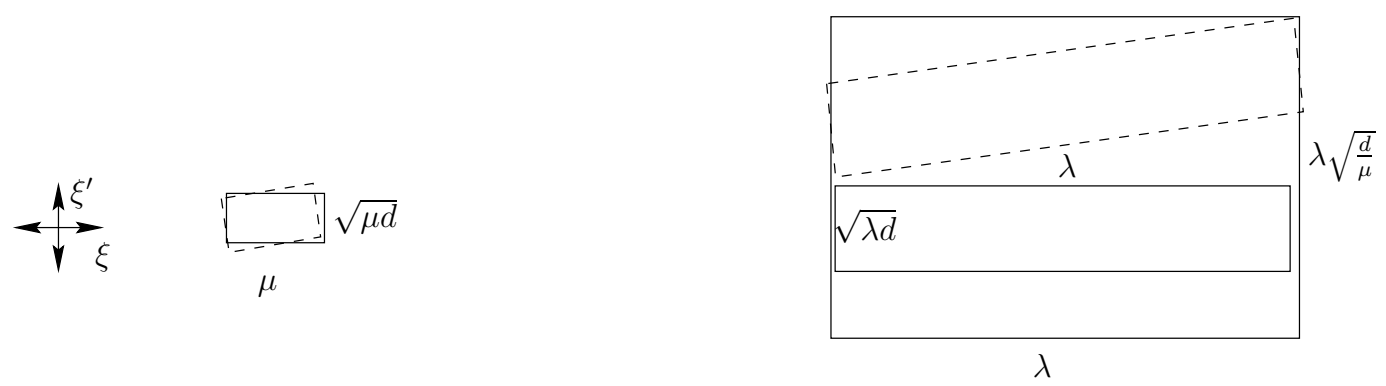

FiguRE 2. Spatial supports in the small angular decomposition.

has $\mu \ll c^{-\frac{1}{2}} \sqrt{\lambda d}$. The result is:

Lemma 8.3 (Small angle decomposition for the term $B$ ). In the ranges stated for the $B$ term above, we can write:

$$
s_{\lambda, d}^{\omega_{1}}\left(s_{\mu} * s_{\lambda, \bullet \leqslant c \mu}\right)=s_{\lambda, d}^{\omega_{1}}\left(s_{\mu} * b_{\lambda,(\lambda d)^{\frac{1}{2}}}^{\omega_{3}} s_{\lambda, \bullet \leqslant c \mu}\right) .
$$

where $\left|\omega_{1}-\omega_{3}\right| \sim \sqrt{\frac{d}{\lambda}}$.

Finally, we note here the important fact that in the decomposition (60) above, the range of interaction in the product forces $d \lesssim \mu$. This completes our list of bilinear decompositions.

\section{Inductive Estimates II: Remainder of the Low $\times$ High $\Rightarrow$ High FREQUENCY INTERACTION}

It remains for us is to bound the term $B$ from line (53) in the $Z_{\lambda}$ space, as well as show the inclusion (50) for the terms $C_{I}-C_{I I I}$ from line (54). We do this now, proceeding in reverse order.

proof of estimate (50) for the $C_{I I I}$ term. To begin with we fix $d$. Using the remarks at the beginning of the proof of (49), we see that it is enough to show that:

$$
\begin{aligned}
\| S_{\lambda, \bullet<\min \{c \mu, d\}}\left(S_{\mu, d} u \nabla S_{\lambda, \bullet<\min \{c \mu, d\}} v\right) & \|_{L^{1}\left(L^{2}\right)} \\
& \lesssim \lambda\left(\sum_{\omega}\left\|S_{\mu, d}^{\omega} u\right\|_{L^{1}\left(L^{\infty}\right)}^{2}\right)^{\frac{1}{2}}\|v\|_{F_{\lambda}} .
\end{aligned}
$$


JACOB STERBENZ

To accomplish this, we first use the wide angle decomposition, (55), on the left hand side of (61). This allows us to compute, using a Cauchy-Schwartz, that:

$$
\begin{aligned}
&\left\|S_{\lambda, \bullet<\min \{c \mu, d\}}\left(S_{\mu, d} u \nabla S_{\lambda, \bullet<\min \{c \mu, d\}} v\right)\right\|_{L^{1}\left(L^{2}\right)}, \\
& \lesssim\left.\sum_{\substack{\omega_{2}, \omega_{3} \\
\left|\omega_{3} \pm \omega_{2}\right| \sim(d / \mu)^{\frac{1}{2}}}}\left\|S_{\mu, d}^{\omega_{2}} u\right\|_{L^{1}\left(L^{\infty}\right)} \cdot \| \nabla B_{\lambda, \lambda\left(\frac{d}{\mu}\right)^{\frac{1}{2}}}^{\omega_{3}} S_{\lambda, \bullet<\min \{c \mu, d\}} v\right) \|_{L^{\infty}\left(L^{2}\right)}, \\
& \lesssim\left.\lambda\left(\sum_{\omega}\left\|S_{\mu, d}^{\omega} u\right\|_{L^{1}\left(L^{\infty}\right)}^{2}\right)^{\frac{1}{2}}\left(\sum_{\omega} \| B_{\lambda, \lambda\left(\frac{d}{\mu}\right) \frac{1}{2}^{\frac{1}{2}}}^{\omega} S_{\lambda, \bullet<\min \{c \mu, d\}} v\right) \|_{L^{\infty}\left(L_{x}^{2}\right)}^{2}\right)^{\frac{1}{2}}, \\
& \lesssim \lambda\left(\sum_{\omega}\left\|S_{\mu, d}^{\omega} u\right\|_{L^{1}\left(L^{\infty}\right)}^{2}\right)^{\frac{1}{2}}\|v\|_{F_{\lambda}} .
\end{aligned}
$$

Summing over $d$ now yields the desired estimate.

proof of (50) for the $C_{I I}$ term. Again, fixing $d$, and using the angular decomposition lemma 8.1 we compute that:

$$
\begin{aligned}
& \left\|S_{\lambda, \bullet<d}\left(S_{\mu, \bullet \leqslant d} u \nabla S_{\lambda, d} v\right)\right\|_{L^{1}\left(L^{2}\right)}, \\
\lesssim & \sum_{\substack{\omega_{2}, \omega_{3} \\
\left|\omega_{3} \pm \omega_{2}\right| \sim(d / \mu)^{\frac{1}{2}}}}\left\|S_{\mu, \bullet \leqslant d}^{\omega_{2}} u\right\|_{L^{2}\left(L^{\infty}\right)} \cdot\left\|B_{\lambda, \lambda\left(\frac{d}{\mu}\right)^{\frac{1}{2}}}^{\omega_{3}} S_{\lambda, d} v\right\|_{L^{2}\left(L^{2}\right)}, \\
\lesssim & \lambda\left(\sum_{\omega}\left\|S_{\mu, \bullet \leqslant d}^{\omega} u\right\|_{L^{2}\left(L^{\infty}\right)}^{2}\right)^{\frac{1}{2}}\left\|S_{\lambda, d} v\right\|_{L^{2}\left(L^{2}\right)}, \\
\lesssim & \lambda \mu^{\frac{n-2}{2}}\left(\frac{d}{\mu}\right)^{\frac{n-5}{4}}\|u\|_{F_{\mu}}\|v\|_{F_{\lambda}} .
\end{aligned}
$$

This last expression can now be summed over $d$, using the condition $d<c \mu$, to obtain the desired result.

proof of (50) for the $C_{I}$ term. This is the other instance where we will have to rely on the $X_{\lambda, 1}^{\frac{1}{2}}$ space. Following the same reasoning used previously, we first bound:

$$
\begin{aligned}
& \left\|\Xi^{-1} S_{\lambda, d}\left(S_{\mu, \bullet \leqslant d} u \nabla S_{\lambda, \bullet \leqslant d} v\right)\right\|_{L^{2}\left(L^{2}\right)}, \\
\lesssim & \left.d^{-1} \sum_{\substack{\omega_{2}, \omega_{3} \\
\left|\omega_{3} \pm \omega_{2}\right| \sim(d / \mu)^{\frac{1}{2}}}}\left\|S_{\mu, \bullet \leqslant d}^{\omega_{2}} u\right\|_{L^{2}\left(L^{\infty}\right)} \cdot \| B_{\lambda, \lambda\left(\frac{d}{\mu}\right)^{\frac{1}{2}}}^{\omega_{3}} S_{\lambda, \bullet \leqslant d} v\right) \|_{L^{\infty}\left(L^{2}\right)}, \\
\lesssim & \left.d^{-1}\left(\sum_{\omega}\left\|S_{\mu, \bullet \leqslant d}^{\omega} u\right\|_{L^{2}\left(L^{\infty}\right)}^{2}\right)^{\frac{1}{2}} \cdot\left(\sum_{\omega} \| B_{\lambda, \lambda\left(\frac{d}{\mu}\right)^{\frac{1}{2}}}^{\omega} S_{\lambda, \bullet \leqslant d} v\right) \|_{L^{\infty}\left(L_{x}^{2}\right)}^{2}\right)^{\frac{1}{2}}, \\
\lesssim & d^{-\frac{1}{2}} \mu^{\frac{n-2}{2}}\left(\frac{d}{\mu}\right)^{\frac{n-5}{4}}\|u\|_{F_{\mu}}\|v\|_{F_{\lambda}} .
\end{aligned}
$$

Multiplying this last expression by $d^{\frac{1}{2}}$ and then using the condition $d<c \mu$ to sum over $d$ yields the desired result for the $X_{\lambda, 1}^{\frac{1}{2}}$ space part of estimate (50). It remains 
to prove the $Z_{\lambda}$ estimate. Here we use the second angular decomposition lemma 8.2 to compute that for fixed $d$ :

$$
\begin{aligned}
& \left(\sum_{\omega_{1}}\left\|\Xi^{-1} S_{\lambda, d}^{\omega_{1}}\left(S_{\mu, \bullet \leqslant d} u \nabla S_{\lambda, \bullet \leqslant d} v\right)\right\|_{L^{1}\left(L^{\infty}\right)}^{2}\right)^{\frac{1}{2}}, \\
& \lesssim(\lambda d)^{-1}\left(\sum_{\substack{\omega_{1}, \omega_{2}, \omega_{3}: \\
\omega_{1}-\omega_{3} \sim(d / \lambda)^{\frac{1}{2}} \\
\omega_{1} \pm \omega_{2} \sim(d / \mu)^{\frac{1}{2}}}} \| S_{\lambda, d}^{\omega_{1}\left(S_{\mu, \bullet \leqslant d}^{\omega_{2}} u \nabla S_{\lambda, \bullet \leqslant d}^{\omega_{3}} v\right) \|_{L^{1}\left(L^{\infty}\right)}^{2}}\right)^{\frac{1}{2}}, \\
& \lesssim d^{-1} \sup _{\omega}\left\|S_{\mu, \bullet \leqslant d}^{\omega} u\right\|_{L^{2}\left(L^{\infty}\right)} \cdot\left(\sum_{\omega}\left\|S_{\lambda, \bullet \leqslant d}^{\omega} v\right\|_{L^{2}\left(L^{\infty}\right)}^{2}\right)^{\frac{1}{2}} \text {, } \\
& \lesssim\left(\frac{d}{\mu}\right)^{\frac{n-5}{4}}\left(\frac{d}{\lambda}\right)^{\frac{n-5}{4}} \mu^{\frac{n-2}{2}} \lambda^{\frac{n-2}{2}}\|u\|_{F_{\mu}}\|v\|_{F_{\lambda}} .
\end{aligned}
$$

Multiplying this last expression by $\lambda^{\frac{2-n}{2}}$ and summing over $d$ using the condition $d<\lambda, \mu$ yields the desired result.

proof of the $Z_{\lambda}$ embedding for the $B$ term. The pattern here follows that of the last few lines of the previous proof. Fixing $d$, we use the decomposition Lemma 8.3 to compute that:

$$
\begin{aligned}
& \left(\sum_{\omega}\left\|\Xi^{-1} S_{\lambda, d}^{\omega}\left(S_{\mu} u \nabla S_{\lambda, \bullet \leqslant c \mu} v\right)\right\|_{L^{1}\left(L^{\infty}\right)}^{2}\right)^{\frac{1}{2}}, \\
& \lesssim(\lambda d)^{-1}\left(\sum_{\substack{\omega_{1}, \omega_{3}: \\
\left|\omega_{1}-\omega_{3}\right| \sim(d / \lambda)^{\frac{1}{2}}}}\left\|S_{\lambda, d}^{\omega_{1}}\left(S_{\mu} u \nabla B_{\lambda,(\lambda d)^{\frac{1}{2}}}^{\omega_{3}} S_{\lambda, \bullet<c \mu} v\right)\right\|_{L^{1}\left(L^{\infty}\right)}^{2}\right)^{\frac{1}{2}}, \\
& \lesssim d^{-1}\left\|S_{\mu} u\right\|_{L^{2}\left(L^{\infty}\right)} \cdot\left(\sum_{\omega}\left\|B_{\lambda,(\lambda d)^{\frac{1}{2}}}^{\omega} S_{\lambda, \bullet \leqslant c \mu} v\right\|_{L^{2}\left(L^{\infty}\right)}^{2}\right)^{\frac{1}{2}} \\
& \lesssim\left(\frac{\mu}{d}\right)^{\frac{1}{2}}\left(\frac{d}{\lambda}\right)^{\frac{n-5}{4}} \mu^{\frac{n-2}{2}} \lambda^{\frac{n-2}{2}}\|u\|_{F_{\mu}}\|v\|_{F_{\lambda}} .
\end{aligned}
$$

Multiplying the last line above by a factor of $\lambda^{\frac{2-n}{2}}$ and using the conditions $d<\lambda$ and $c \mu<d \lesssim \mu$, we may sum over $d$ to yield the desired result.

\section{REFERENCES}

[1] Nikolaos Bournaveas Local existence for the Maxwell-Dirac equations in three space dimensions. Comm. Partial Differential Equations 21 (1996), no. 5-6, 693-720.

[2] Damiano Foschi, Sergiu Klainerman Bilinear space-time estimates for homogeneous wave equations. Ann. Sci. cole Norm. Sup. (4) 33 (2000), no. 2, 211-274. 
[3] Markus Keel, Terence Tao Endpoint Strichartz estimates. Amer. J. Math. 120 (1998), no. 5, 955-980.

[4] Sergiu Klainerman Uniform decay estimates and the Lorentz invariance of the classical wave equation. Comm. Pure Appl. Math. 38 (1985), no. 3, 321-332.

[5] Sergiu Klainerman, Matei Machedon Space-time estimates for null forms and the local existence theorem. Comm. Pure Appl. Math. 46 (1993), no. 9, 1221-1268.

[6] Sergiu Klainerman, Matei Machedon Smoothing estimates for null forms and applications. Duke Math Journal 81 (1995), no. 1, pp. 99-103.

[7] Sergiu Klainerman, Matei Machedon Estimates for null forms and the spaces $H^{s, \delta}$. Internat. Math. Res. Notices 1996, no. 17, 853-865.

[8] Sergiu Klainerman, Daniel Tataru On the optimal local regularity for Yang-Mills equations in $R^{4+1}$. J. Amer. Math. Soc. 12 (1999), no. 1, 93-116.

[9] Sergiu Klainerman, Sigumd Selberg, Bilinear estimates and applications to nonlinear wave equations. Commun. Contemp. Math. 4 (2002), no. 2, 223-295.

[10] Hans Lindblad Counterexamples to local existence for semi-linear wave equations. Amer. J. Math. 118 (1996), no. 1, 1-16.

[11] Igor Rodnianski, Terence Tao Global regularity for the Maxwell-Klein-Gordon equation in high dimensions. preprint

[12] Terence Tao Global regularity of wave maps. I. Small critical Sobolev norm in high dimension. Internat. Math. Res. Notices 2001, no. 6, 299-328.

[13] Daniel Tataru Local and global results for wave maps I. Comm. Partial Differential Equations 23 (1998), no. 9-10, 1781-1793

[14] Daniel Tataru On global existence and scattering for the wave maps equation. Amer. J. Math. 123 (2001), no. $1,37-77$

[15] Daniel Tataru On the equation $\square u=|\nabla u|^{2}$ in $5+1$ dimensions. Math. Res. Lett. 6 (1999), no. 5-6, 469-485.

Department of Mathematics, Princeton University, Princeton NJ, 08544

E-mail address: sterbenz@math.princeton.edu 